\title{
LESS PRECISE REPRESENTATION OF NUMERICAL MAGNITUDE IN HIGH MATH-ANXIOUS INDIVIDUALS: AN ERP STUDY OF THE SIZE AND DISTANCE EFFECTS
}

\author{
M. Isabel Núñez-Peña ${ }^{1,2}$ and Macarena Suárez-Pellicioni ${ }^{1,2}$
}

1 Department of Behavioral Sciences Methods, Faculty of Psychology, University of Barcelona, Spain

2 Institute for Brain, Cognition, and Behavior (IR3C), University of Barcelona, Spain

\section{Correspondence to:}

Maria Isabel Núñez-Peña

Department of Behavioral Sciences Methods

Faculty of Psychology

University of Barcelona

Passeig Vall d’Hebron, 171

08035 Barcelona (SPAIN)

Tel. +34 933125853

Fax: +34 934021359

E-mail: inunez@ub.edu

Citation: Núñez-Peña, M.I. \& Suárez-Pellicioni, M. (2014). Less precise representation of numerical magnitude in high math anxious individuals: An ERP study of the size and distance effects. Biological Psychology, 103, 176-183. 


\section{Support:}

This research was supported by the Spanish Ministry of Economy and Competitiveness under grant PSI2012-35703, the Spanish Ministry of Science and Technology under grant BES-2010-036859, and the Generalitat de Catalunya under grant SGR2014-177. 


\begin{abstract}
Numerical comparison tasks are widely used to study the mental representation of numerical magnitude. In the present study, event-related brain potentials (ERPs) were recorded while 26 high math-anxious (HMA) and 27 low math-anxious (LMA) individuals performed a numerical comparison task, our aim being to examine whether HMA individuals present a poorer mental representation of numerical magnitude than do their LMA peers. Participants were presented with pairs of single-digit Arabic numbers and were asked to decide which one had the larger numerical magnitude. The size of the numbers and the distance between them were manipulated in order to study the size and the distance effects. The results showed that HMA individuals were slower than their LMA peers but did not differ in hit rate, suggesting that in order to gain accuracy the HMA group lost speed. Moreover, both distance and size effects were larger for the HMA group. As for ERPs, results showed that the ERP distance effect had larger amplitude for both the size and distance effects in the HMA group than among their LMA counterparts. Since this component has been taken as a marker of the processing of numerical magnitude, this result suggests that HMA individuals have a less precise representation of numerical magnitude, which could explain their poorer performance in mathematical tasks.
\end{abstract}

Key Words: math anxiety; distance effect; size effect; ERPs; numerical number line 


\section{INTRODUCTION}

The task of comparing a pair of numbers is subject to two highly robust phenomena, the distance effect and the size effect, which were first described in Moyer and Landauer's (1967) seminal paper. The distance effect refers to the fact that it is easier to compare two numbers (i.e., telling which is the largest or the smallest) if they are far apart than if they are close (e.g., a comparison of 1 and 9 will be faster and less error prone than a comparison of 8 and 9). The size effect refers to the fact that a comparison of two numbers which are equated for numerical distance is more difficult for large numbers than for small numbers (e.g., a comparison of 8 and 9 will be slower and more error prone than a comparison of 1 and 2). Both effects occur with numbers presented in Arabic format (Dehaene, Dupoux, \& Mehler, 1990), verbal format (Koechlin, Naccache, Block, \& Dehaene, 1999), and nonsymbolic format (Buckley \& Gillman, 1974), and they have been observed in humans, even early in childhood (Duncan \& McFarland, 1980), as well as in animals (Dehaene \& Changeux, 1993).

The distance and size effects are attributed to the access to the mental number line along which the numerical magnitudes are represented. To account for the distance effect it has been suggested that close magnitudes are represented in the mental number line with overlapping distributions of activation and, therefore, they are more difficult to discriminate than are distant magnitudes (Restle, 1970; Libertus \& Brannon, 2010). The activation pattern of each numerical magnitude is proposed to follow a Gaussian distribution that peaks at the target magnitude and decreases with increasing distance from the target. Thus, magnitudes that are numerically closer to each other will have more representational overlap than will magnitudes that are numerically farther apart, and, as a consequence, the former will produce slower and less accurate responses than will the latter. To account for 
the size effect it has been suggested that large numbers are represented in the mental number line more vaguely than are small numbers and, hence, it is more difficult to discriminate between larger numbers than between smaller numbers when the numerical distance between them is equal (Antell \& Keating, 1983; Strauss \& Curtis, 1981). In other words, the standard deviation of the Gaussian distribution of each magnitude increases as number size increases and, therefore, the activation distributions overlap more for larger numerical magnitudes than for smaller ones. Since the distance and size effects are related to the access to the numerical magnitude representation in the mental number line, they have usually been used as a behavioral measure of the precision of numerical estimation.

Recently, Maloney, Ansari, and Fugelsang (2011) studied the precision of numerical estimation in high and low math-anxious individuals. Math anxiety is defined as a negative reaction to math and to mathematical situations that is negatively related to math achievement or competence (Ashcraft \& Ridley, 2005). In their Experiment 1, Maloney et al. (2011) used a number comparison task with a fixed standard (i.e., telling whether a single-digit Arabic number was larger or smaller than 5), while in their Experiment 2 they used a simultaneous number comparison task (i.e., telling which of two single-digit Arabic numbers presented simultaneously had larger numerical magnitude). In both experiments they found that the distance effect was greater for the high math-anxious group than for the low math-anxious group (this effect was found for response time but not for accuracy), leading them to conclude that high math-anxious individuals suffer from a low-level numerical deficit that compromises the development of more complex mathematical skills (see also, Maloney, Risko, Ansari, \& Fugelsang, 2010). This conclusion is based on the fact that the size of the distance effect is related to differences in math achievement: the smaller the distance effect, the higher the level of mathematical skill (De Smedt, Verschaffel, \& 
Ghesquière, 2009; Holloway \& Ansari, 2009). Maloney et al.'s results are important because they cast some doubts on the explanation proposed by Ashcraft and colleagues (e.g., Ashcraft \& Kirk, 2001; Ashcraft, Kirk, \& Hopko, 1998; Ashcraft \& Krause, 2007) for why math anxiety affects math performance.

According to Ashcraft and colleagues, math anxiety consumes working memory resources and, as a consequence, reduces the resources necessary to solve complex math problems (i.e., those that require working memory resources in order to be performed). Ashcraft and colleagues' proposal is based on two premises. First, math anxiety is related to performance in complex arithmetical problems (e.g., multi-digit additions that require working memory resources in order to be solved) but not to performance in simple arithmetical problems (e.g., single-digit additions) (anxiety-complexity effect; Ashcraft \& Faust, 1994). And second, in accordance with the theory of Eysenck and Calvo (Eysenck, 1992, 1997; Eysenck \& Calvo, 1992), anxiety produces intrusive thinking that consumes working memory resources. Thus, if math-anxious individuals are presented with mathematical problems that require working memory resources in order to be performed, they will have fewer working memory resources available because some of them will be occupied with intrusive thinking generated by their high level of anxiety, the consequence being that their performance on these problems will be poorer. Distinguishing between the proposal of Maloney and colleagues (i.e., basic numerical deficit in math-anxious individuals) and that of Ashcraft and colleagues (i.e., anxiety affecting working memory through intrusive thinking) is relevant because understanding the cognitive determinants of math anxiety is necessary in order to design appropriate interventions that can prevent math anxiety effects on math performance. 
The present study was designed to investigate the number magnitude representation in high and low math-anxious individuals. It differed from previous investigations in two ways. First, the size effect, in addition to the distance effect, was studied. Second, we used event-related brain potentials (ERPs) to address the question of whether high and low mathanxious individuals differ in their processing of numerical magnitude, such that the former show a deficit in the approximate calculation system, as Maloney et al. (2011) have suggested.

Recent research has reported a link between the distance effect and neurophysiological signatures revealed through ERPs. First, a positive peak, with latency around 200 ms poststimulus and maximum amplitude over posterior electrode sites, has been reported in comparison tasks with a fixed standard (e.g., compare the presented number with 5: Dehaene, 1996; Temple \& Posner, 1998; Libertus, Woldorff, \& Brannon, 2007; compare the number with 15: Turconi, 2004; or compare the number with 65: Pinel, Dehaene, Rivière, \& LeBihan, 2001). This component, known as P2p, is sensitive to the distance between the two numbers to be compared: the smaller the distance, the greater the positivity. It has been reported in both symbolic (Arabic numerals and written number words: Dehaene, 1996; Libertus et al., 2007; Temple \& Posner, 1998) and nonsymbolic comparison tasks (dot patterns: Dehaene, 1996; Libertus et al., 2007; Smets, Gebuis, \& Reynvoet, 2013; Temple \& Posner, 1998), as well as in passive viewing tasks in an adaptation context (Hyde \& Spelke, 2008, 2012; Hyde \& Wood, 2011). Second, in simultaneous number comparison tasks (i.e., telling which of two simultaneously presented numbers has the larger numerical magnitude), a positive peak with latency around $200 \mathrm{~ms}$ post-stimulus has also been found This ERP distance effect also shows greater positivity when the distance between the two numbers to be compared decreases. However, its scalp 
distribution is less clear than that of the P2p. There are reports of an ERP distance effect with diffuse topography (Szücs \& Soltész, 2008) or with a fronto-central distribution (Szücs \& Soltész, 2007). The link between the distance effect and these early ERP components makes them good markers of approximate numerical magnitude processing (Hyde \& Wood, 2011), and they could be useful instruments for shedding more light on whether math anxiety is related to a deficit in elementary numerical cognition.

In the present study, high and low math-anxious individuals were asked to perform a simultaneous comparison task. They were presented with pairs of single-digit Arabic numerals and were required to decide which number had the larger magnitude. The size of the numbers and the numerical distance between them were manipulated in order to study the size and distance effects. The simultaneous comparison task was used instead of the 'comparison with five' task because the latter would only have allowed us to study the distance effect. Moreover, there are two more reasons to prefer the simultaneous comparison task. First, according to Maloney el al. (2011) it is possible that the 'comparison with five' task requires more working memory resources than the simultaneous comparison task, which would make it difficult to rule out the possibility that working memory plays a role in the results. Second, Maloney, Risko, Preston, Ansari, and Fugelsang (2010) demonstrated that whereas the symbolic simultaneous comparison task is reliable in response time, the 'comparison with five' task is not (as for errors, reliability indexes were marginally significant in both tasks).

In the present study, groups were formed in such a way that they differed in math anxiety but not in trait anxiety, thereby ruling out the possibility that the latter factor might account for any differences between groups. Behavioral and electrophysiological measures were recorded. Based on previous studies our predictions were as follows. In terms of 
response time, if math-anxious individuals have a less precise representation of magnitude they should present not only a larger distance effect than do their low math-anxious peers (as previously reported by Maloney et al., 2011) but also a larger size effect. We did not expect differences between groups in hit rate. As for ERPs, if math anxious individuals have a less precise representation of magnitude they should show a larger ERP distance effect than do their low math-anxious peers. We also sought to examine whether a similar ERP effect would be found for the size effect.

\section{METHODS}

\subsection{Participants}

Fifty-three healthy volunteers were tested in this study, 26 with a high level of math anxiety and 27 with a low level. They were selected from among a sample of 629 students from the University of Barcelona who were assessed for math anxiety, trait anxiety, and arithmetic ability (see Material).

The low math-anxious group (henceforth, LMA) comprised 27 participants (age range $=19-31$, mean $=21.59, \operatorname{SEM}=.63$ ) who scored below the first quartile on the Shortened Mathematics Anxiety Rating Scale (sMARS) (Alexander \& Martray, 1989) (score range $=30-56, \quad$ mean $=44.26, \quad S E M=1.38$ ). The high math-anxious group (henceforth, HMA) comprised 26 participants (age range $=19-25$, mean $=20.92$, SEM $=.30$ ) who scored above the third quartile on the sMARS (score range $=75-102$, mean $=85.08, \mathrm{SEM}=1.25)$. More detailed information about the two groups is shown in Table 1.

Groups differed in math anxiety $(t(51)=21.805, p<.001)$ but not in trait anxiety $(t(51)=1.252, \quad p=.216), \quad$ age $(t(51)=.938, \quad p=.353)$, years of formal education 
$(t(51)=.022, p=.983)$, gender distribution ${ }^{1}\left(\chi^{2}=3.592, p=.058\right)$, handedness $\left(\chi^{2}=1.165\right.$, $p=.280)$, or ethnicity $\left(\chi^{2}=1.058, p=.304\right)$. Groups did not differ in arithmetic ability when the number of correctly solved operations over the total number of operations attempted by the participant on the Addition test from the French kit was taken as an indicator of math ability $(t(30)=.658, p=.515)$. However, differences between groups were found when the number of correctly solved operations over the total number of operations included in the Addition test was used $(t(30)=2.093, p=.046)$.

All had normal or corrected-to-normal visual acuity and they did not report any history of neurological or psychiatric disorders. Participants were naïve as to the purposes of the study.

INSERT TABLE 1 ABOUT HERE

Participants were paid for their participation and gave written informed consent before the experiment. The experimental protocol was approved by the Ethics Committee of the University of Barcelona and was in accordance with the Code of Ethics of the World Medical Association (Declaration of Helsinki).

\subsection{Material}

Groups were formed according to the participants's scores on the following tests:

\footnotetext{
${ }^{1}$ An additional analysis showed that men and women did not differ in either the distance effect $(t(51)=.474$, $p=.638)$ or the size effect $(\mathrm{t}(51)=.742, \mathrm{p}=.462)$, both measured in terms of response time; therefore, gender cannot explain the differences we found between the two math-anxious groups. It is worth mentioning that although females usually report higher levels of math anxiety than do males, this result contrasts with the fact that female students typically obtain similar, or only slightly lower, levels of achievement in mathematics relative to their male peers. As stated by Goetz, Bieg, Lüdtke, Pekrun, and Hall (2013), girls' competence beliefs could be partly responsible for their higher levels of reported math anxiety. In other words, females may not experience greater anxiety than males, but they may tend to overestimate their level of math anxiety.
} 
Shortened Mathematics Anxiety Rating Scale (sMARS) (Alexander \& Martray, 1989). The sMARS is a 25-item version of the Math Anxiety Rating Scale (MARS) (Richardson \& Suinn, 1972). This instrument measures anxiety by presenting respondents with 25 situations which may cause math anxiety (e.g., Being given a homework assignment of many difficult problems which is due the next class meeting). Respondents indicate the level of anxiety associated with the item on a five-point Likert scale from 1 (no anxiety) to 5 (high anxiety). The sum of the item scores provides the total score for the instrument, which ranges from 25 to 125 . In the present study, we used the Spanish version of the sMARS (Núñez-Peña, Suárez-Pellicioni, Guilera, \& Mercadé-Carranza, 2013), whose scores have shown strong internal consistency (Cronbach’s alpha $=.94)$ and high 7-week test-retest reliability (intra-class correlation coefficient $=.72$ ).

State-Trait Anxiety Inventory (STAI) (Spielberger, Gorsuch, Lushene, Vagg, \& Jacobs, 1983). The STAI is a 40-item scale used to measure state (STAI-S) and trait (STAI-T) anxiety. This study only considered the STAI-T score, which reflects a more general and relatively stable tendency to respond with anxiety. This subscale includes 20 statements describing different emotions, and respondents must answer by considering how they feel “in general”. Items are answered on a four-point Likert scale, from 0 (almost never) to 3 (almost always). Good to excellent internal consistency (Cronbach's alpha $=.95$ ), adequate 30-day test-retest reliability with high school students $(r=.75)$, and 20-day test-retest reliability with college students $(r=.86)$ have been reported for the Spanish version of this subscale (Spielberger, Gorsuch, \& Lushene, 2008).

Addition test from the French kit (French, Ekstrom, \& Price, 1963). This test consists of 60 additions involving three numbers of either one or two digits (e.g., $6+67+38$ ), presented vertically. Participants were asked to solve the additions as fast and as accurately 
as possible during a 2-min period. Two measures of participants' arithmetical ability were calculated. First, the number of correctly solved operations over the total number of operations attempted by the participant (i.e., a measure of precision) and, second, the number of correctly solved operations over the total number of operations in the test (i.e., a measure of speed).

A number comparison task was presented to each participant during the recording session. The stimuli consisted of a pair of numbers shown simultaneously at the center of a computer screen. The number size effect was studied by the pairs "1 2 " (small size) and "8 9” (large size), while the distance effect was studied using the pairs “1 8” and “2 9” (distance 7) and “8 9" (distance 1). These pairs were chosen in order to be as extreme as possible over single-digit numbers. Numbers were presented in font size 60 (Courier New) and subtended view angles of $1.03^{\circ}$ (horizontally) and $1.43^{\circ}$ (vertically). There were two instances for all number pairs (e.g., “1 2 ” and “2 1 ”) and all pairs were presented an equal number of times.

\subsection{Procedure}

Participants were tested individually. Upon entering the experimental room they completed standard procedures concerning informed consent, along with a demographics questionnaire enquiring about their age, ethnicity, gender, and number of years of formal education. EEG/EOG sensor electrodes were then attached and the participant was given detailed task instructions. Next, participants were seated $100 \mathrm{~cm}$ away from the computer screen in an electrically-shielded, sound-attenuating recording chamber. The experimental session began with a training period of 16 trials. The training trials were only used to familiarize the participants with the task, and they were not included in the statistical 
analysis. The experiment, including electrode placement and practice and recording sessions, lasted about an hour.

The task for participants consisted in indicating the number of larger numerical magnitude by pressing the left or right button of the mouse, depending on the side of the screen in which the number of larger numerical magnitude had appeared. For example, for the pair of numbers “ 12 ”, participants had to click the right button of the mouse, given that the number of greatest numerical magnitude (in this case, 2) appeared on the right side of the screen. Participants were encouraged to respond as fast as possible. Each trial began with a fixation sign (an asterisk) shown for $500 \mathrm{~ms}$. After a $300 \mathrm{~ms}$ pause a pair of numbers were shown for 300 ms, this being followed by a 700 ms black screen (maximum response windows of $1000 \mathrm{~ms})^{2}$. Each trial was followed by a variable inter-trial interval ranging from 600 to $1100 \mathrm{~ms}$. The recording session consisted of four blocks of 40 stimuli (total of 160 trials) and there was a 1-minute pause between blocks. Trials were randomly presented to each participant.

The presentation of stimuli and the collection of behavioral data were controlled by EPrime 2.0 software (Psychology Software Tools Inc., Sharpsburg, PA, USA).

\subsection{Electrophysiological recording}

The EEG was recorded using ANT hardware and software (B.V., Enschede, The Netherlands) from 64 electrodes positioned according to the extended 10/20 system, as well as two electrodes on the right and left mastoids, all mounted in a commercial WaveGuard

\footnotetext{
2 Means of percentages of timeouts across conditions were 0.37 (SEM = 0.17) for small size, $0.61(\mathrm{SEM}=$ $0.29)$ for large size and $0.32(\mathrm{SEM}=0.14)$ for distance 7 conditions. There were no difference between conditions on the amount of percentages of timeouts, $F(2,104)=1.26, p=.28$.
} 
EEG Cap (Eemagine Medical Imaging Solutions GmbH. ANT Advanced Neuro Technology). EEG channels were continuously digitized at a rate of $512 \mathrm{~Hz}$ by an ANT amplifier (B.V., Enschede, The Netherlands). A band-pass filter was set from 0.16 to 30 $\mathrm{Hz}$, and electrode impedance was kept below $5 \mathrm{k} \Omega$. The horizontal and vertical electrooculogram was recorded with electrodes placed at the outer canthus and below the right eye respectively. The common reference electrode was placed on the tip of the nose and ground was located at AFz.

\subsection{Data analysis}

Analyses were performed separately for the size effect and the distance effect. In order to study the size effect we first analyzed response time (RT) for correctly solved trials and the percentage of correct responses with analyses of variances (ANOVAs), taking Size (small, large) as the within-subject factor and Group (LMA, HMA) as the between-subjects factor. For RT analysis the recursive outlier removal procedure (Van Selst \& Jolicoeur, 1994) was used in order to control for the effects of outliers. ERP data were then analyzed. Two ERP averages were calculated per participant: one for small number trials and one for large number trials. The averages were constructed from -200 ms to $600 \mathrm{~ms}$ epochs relative to stimulus onset. Trials with voltages exceeding 20 standard deviations in the EOG electrodes and $\pm 100 \mu \mathrm{V}$ in the remaining electrodes were excluded from the ERP average. Ocular artifacts were identified and corrected with the eye-movement correction algorithm used in the EEprobe program (ANT, The Netherlands). Three participants were excluded from the analysis due to technical problems. An ANOVA was performed on the ERP mean amplitude in the 200-250 ms window in order to study the size effect. Analysis was performed at nine electrodes (F3, Fz, F4, C3, Cz, C4, P3, Pz, P4), taking Size (small, large), 
Frontality (frontal, central, parietal), and Laterality (three levels from left to right) as within-subject factors and Group (LMA, HMA) as the between-subjects factor. In the ANOVAs the $F$ value, the uncorrected degrees of freedom, the probability level following correction, the $\varepsilon$ value (when appropriate), and the $\eta_{p}^{2}$ effect size index are given. Whenever an interaction reached significance, simple effect analyses were performed and the Hochberg approach was used to control for the increase in Type I error (Keselman, 1998). Only significant effects $(p \leq .05)$ are reported.

As for the distance effect, the analysis performed was identical to that described for the size effect, except that the within-subject factor in the ANOVAs was Distance (distance 1, distance 7) rather than Size. Only the large number trials (i.e., "8 9”) were used for distance 1 in order to avoid confusing the distance effect with the size effect.

\section{DATA ANALYSIS AND RESULTS}

\subsection{Size effect}

\subsubsection{Behavioral data}

Regarding response times, trials with small numbers were solved faster (344 ms) than were those with large numbers (380 ms), $F(1,51)=51.498, p<.001, \eta_{p}^{2}=.502$, thereby showing the well-known size effect. Moreover, HMA individuals were slower (376 ms) than their LMA counterparts (348 ms), $F(1,51)=4.419, p=.041, \eta_{p}^{2}=.080$. The Size $\mathrm{x}$ Group interaction was marginally significant, $F(1,51)=3.531, p=.066, \eta_{p}^{2}=.065$, showing that the size effect (large-small difference) was larger in the HMA group (46 ms) than in the LMA group (26 ms), $t(51)=1.879, p=.066$. 
Regarding the percentage of correct responses, more hits were made in trials with small numbers $(94 \%)$ than in those with large numbers $(76 \%), F(1,51)=150.852, p<.001$, $\eta_{p}{ }^{2}=.747$. Neither the main effect of Group nor the Size $x$ Group interaction reached significance. Means and standard errors for response time and hit rate for the small and large size conditions in the LMA and HMA groups are given in Table 2.

\section{INSERT TABLE 2 ABOUT HERE}

\subsubsection{Event-related potentials}

Figure 1A shows difference waves (large-small number trials) for the HMA and LMA groups at Fz, Cz, and Pz. It can be seen that the size effect elicits a positive wave peaking in the 200-250 ms window, with larger amplitude in the HMA group than in the LMA group at Fz and Cz. Scalp topographic maps in Figure 1B show the size effect on brain activity in the 200-250 ms window for both groups. This figure reveals that the positive component is larger at fronto-central positions in the HMA group as compared with the LMA group. Topographic maps were plotted using the EEProbe 3.1 program (ANT Software BV, Enschede, The Netherlands).

INSERT FIGURE 1 ABOUT HERE

The main effect of Size $\left(F(1,48)=26.031, p<.001, \eta_{p}^{2}=.352\right)$ and, more interestingly, the Size $x$ Group $x$ Frontality interaction $\left(F(2,96)=4.235, p=.033, \varepsilon=.659, \eta_{p}^{2}=.081\right)$ were statistically significant. Separate ANOVAs were performed at frontal, central, and 
parietal sites in order to study this interaction in more detail, taking Size and Laterality as within-subject factors and Group as the between-subjects factor. The main effect of Size reached significance at frontal $\left(F(1,48)=26.652, \quad p<.001, \quad \eta_{p}^{2}=.357\right)$, central $\left(F(1,48)=27.877, p<.001, \eta_{p}^{2}=.367\right)$, and parietal sites $(F(1,48)=13.356, p=.001$, $\eta_{p}{ }^{2}=.218$ ), showing that the amplitude was more positive for large number trials than for small number trials (2.32 $\mu \mathrm{V}$ vs. $.97 \mu \mathrm{V}$ at frontal sites; $2.08 \mu \mathrm{V} v$ s. .51 $\mu \mathrm{V}$ at central sites; and $1.77 \mu \mathrm{V}$ vs. .64 $\mu \mathrm{V}$ at parietal sites). The Size $x$ Group interaction was significant at frontal sites $\left(F(1,48)=7.549, p=.008, \eta_{p}{ }^{2}=.136\right)$ and marginally significant at central sites $\left(F(1,48)=3.324, p=.075, \eta_{p}^{2}=.065\right)$. In order to study these interactions separate ANOVAs were performed for each group, taking Size and Laterality as within-subject factors. Results showed that whereas voltage was more positive for large number than for small number trials in both groups at central $\left(F(1,24)=33.383, p<.001, \eta_{p}{ }^{2}=.582\right.$ and $F(1,24)=4.801, p=.038, \eta_{p}^{2}=.167$ for HMA and LMA, respectively) and parietal sites $\left(F(1,24)=5.115, p=.033, \eta_{p}^{2}=.178\right.$ and $F(1,48)=8.471, p=.008, \eta_{p}^{2}=.261$ for LMA and HMA, respectively), this difference was only significant for the HMA group at frontal sites $\left(F(1,24)=45.843, p<.001, \eta_{p}^{2}=.656\right)$.

When the size effect (large-small difference) was analyzed taking Frontality and Laterality as within-subject factors and Group as the between-subjects factor, the Group x Frontality interaction was significant $\left(F(2,96)=4.235, p=.033, \varepsilon=.659, \eta_{p}{ }^{2}=.081\right)$. The size effect was analyzed for each level of frontality, taking Laterality as the within-subject factor and Group as the between-subjects factor. The Group effect was significant at frontal sites $\left(F(1,48)=7.549, p=.008, \quad \eta_{p}^{2}=.136\right)$, the size effect being higher in HMA individuals than in LMA individuals (2.08 $\mu \mathrm{V}$ vs. .63 $\mu \mathrm{V}$, respectively). At central sites the 
Group effect was close to significance $\left(F(1,48)=3.324, p=.075, \eta_{p}^{2}=.065\right)$, showing a similar pattern to that reported for frontal sites $(2.12 \mu \mathrm{V} v$ s. $1.03 \mu \mathrm{V}$ for HMA and LMA individuals, respectively).

\subsection{Distance effect}

\subsubsection{Behavioral data}

As for response time, distance- 1 trials were solved slower (380 ms) than were distance-7 trials (333 ms), $F(1,51)=74.302, p<.001, \eta_{p}^{2}=.593$, thereby showing the well-known distance effect. Moreover, HMA individuals were slower (370 ms) than their LMA counterparts $(343 \mathrm{~ms}), F(1,51)=4.639, p=.036, \eta_{p}^{2}=.083$. The Distance $\mathrm{x}$ Group interaction was marginally significant, $F(1,51)=3.812, p=.056, \eta_{p}{ }^{2}=.070$, showing that the distance effect (distance 1-distance 7 difference) was larger in the HMA group (58 ms) than in the LMA group (37 ms), $t(51)=1.952, p=.056$.

Regarding the percentage of correct responses, more hits were made in distance-7 trials (96\%) than in distance-1 trials (76\%), $F(1,51)=150.852, p<.001, \eta_{p}^{2}=.747$. Neither the main effect of Group nor the Size x Group interaction reached significance. Means and standard errors for response time and hit rate for the distance- 1 and distance-7 conditions in the LMA and HMA groups are given in Table 3.

INSERT TABLE 3 ABOUT HERE

\subsubsection{Event-related potentials}


Figure 2A shows difference waves (distance 1-distance 7) for the HMA and LMA groups at Fz, Cz, and Pz. This figure reveals a positive wave peaking at about 200-250 ms post-stimulus that has larger amplitude in HMA individuals than in their LMA peers. Topographic maps in Figure 2B show the distance effect on brain activity in the 200-250 ms window for both groups. It can be seen that the HMA group shows greater positivity at fronto-central positions than do their LMA counterparts.

\section{INSERT FIGURE 2 ABOUT HERE}

The overall ANOVA revealed that the interactions Distance x Group $(F(1,48)=12.388$, $\left.p=.001, \eta_{p}^{2}=.205\right)$ and Distance $\mathrm{x}$ Group $\mathrm{x}$ Laterality $(F(2,96)=4.277, p=.021$, $\varepsilon=.888, \eta_{p}^{2}=.082$ ) were statistically significant. In order to study these interactions in more detail, separate ANOVAs were performed at right, central, and left positions, taking Distance and Frontality as within-subject factors and Group as the between-subjects factor. The Distance $\mathrm{x}$ Group interaction was significant at all the positions analyzed $\left(F(1,48)=8.972, \quad p=.004, \quad \eta_{p}^{2}=.157\right.$ at right positions; $F(1,48)=14.584, p<.001$, $\eta_{p}{ }^{2}=.233$ at left positions; and $F(1,48)=12.066, p=.001, \eta_{p}{ }^{2}=.201$ at central positions). Separate ANOVAs for each group, taking Distance and Laterality as within-subject factors, revealed that voltage was more positive for distance- 1 than for distance-7 only for HMA individuals at left $\left(F(1,24)=18.879, p<.001, \eta_{p}^{2}=.440 ; 1.44 \mu \mathrm{V}\right.$ vs. $4.42 \mu \mathrm{V}$ for distance-7 and distance-1, respectively), right $\left(F(1,24)=17.178, p<.001, \eta_{p}^{2}=.417 ; 1.69\right.$ $\mu \mathrm{V}$ vs. $2.69 \mu \mathrm{V}$ for distance-7 and distance-1, respectively), and central positions, 
$\left(F(1,24)=17.830, p<.001, \eta_{p}^{2}=.426 ; 1.4 \mu \mathrm{V}\right.$ vs. $2.69 \mu \mathrm{V}$ for distance-7 and distance-1, respectively).

When the distance effect (distance 1-distance 7 differences) was analyzed by means of an ANOVA, taking Frontality and Laterality as within-subject factors and Group as the between-subjects factor, the Group effect $\left(F(1,48)=12.388, p=.001, \eta_{p}^{2}=.205\right)$ and the Group x Laterality interaction $\left(F(2,96)=4.277, p=.021, \varepsilon=.888, \eta_{p}^{2}=.082\right)$ were significant. To study this interaction in more detail, separate ANOVAs were performed for each level of laterality, taking Frontality as the within-subject factor and Group as the between-subjects factor. Results showed that voltage was more positive for HMA individuals than for their LMA counterparts at left $\left(F(1,48)=14.584, p<.001, \eta_{p}{ }^{2}=.233\right.$; -.69 $\mu \mathrm{V}$ vs. .98 $\mu \mathrm{V}$ for LMA and HMA, respectively), right $(F(1,48)=8.972, p=.004$, $\eta_{p}^{2}=.157 ;-.24 \mu \mathrm{V}$ vs. .1.01 $\mu \mathrm{V}$ for LMA and HMA, respectively), and central positions $\left(F(1,48)=12.066, p=.001, \quad \eta_{p}^{2}=.201 ;-.46 \mu \mathrm{V}\right.$ vs. $1.25 \mu \mathrm{V}$ for LMA and HMA, respectively).

\section{DISCUSSION}

Our central aim in this study was to examine whether high math-anxious individuals present a poorer mental representation of numerical magnitude than do their low mathanxious counterparts. To this end, ERPs were recorded while HMA and LMA individuals performed a number comparison task, with the numerical distance and size effects being analyzed. These effects were used to address the research question because they provide measures for indexing the representation of numerical magnitude, both behaviorally and 
psychophysiologically. Consequently, they are especially useful in terms of shedding more light on whether math anxiety is related to a deficit in elementary numerical cognition.

As for behavioral measures, our results confirmed our predictions. HMA individuals were slower than their LMA peers, although the groups did not differ in hit rate. The same result was reported in the study by Maloney et al. (2011) and demonstrates that to achieve the level of accuracy shown by the LMA group, HMA individuals need to become slower in their responses ${ }^{3}$. This result agrees with what we found in the analysis of data from the Addition test from the French kit, which showed that groups did not differ in the number of correctly solved operations over the total number of operations attempted by each participant, but they did differ when we analyzed the number of correctly solved operations over the total number of operations included in the test. Therefore, the two groups did not differ in the precision of their responses, but HMA participants solved fewer operations than did their LMA counterparts in the 2-minute period of time they had available. The results of the present experiment also provide converging evidence for the fact that math anxiety effects can be found even in very basic numerical processing tasks (e.g., a numerical comparison task), where working memory resources do not need to be employed. This questions the proposal of Ashcraft and colleagues (e.g., Ashcraft \& Kirk, 2001; Ashcraft, Kirk, \& Hopko, 1998; Ashcraft \& Krause, 2007), as according to them "a mathanxious individual's performance in a math task would be expected to deteriorate to the extent that the task arouses anxiety, but only if the task depends on substantial working memory processing” (Ashcraft \& Ridley, 2005, p. 322). As Maloney et al. (2011) suggested, a hybrid theory can best explain the relationship between high math anxiety and

\footnotetext{
${ }^{3}$ Participants in the present experiment were faster than participants in Maloney et al.'s experiment (2011). This was probably due to the fact that we instructed them to respond quickly. This might also explain the high error rate we found.
} 
a low level of math achievement. They suggested that high math-anxious individuals suffer from a low level numerical deficit that would be at the base of their difficulties with more complex mathematics. These math difficulties, in turn, would result in WM-demanding ruminations when they perform math tasks, which would exacerbate the initial difficulties they experienced.

Our behavioral results also showed robust distance and size effects. First, response time increased and hit rate decreased when the distance between the two Arabic numerals to be compared increased. And second, when the distance between the two Arabic numerals was equal, response time was slower and accuracy decreased for larger numbers. Concerning the Distance x Group and Size x Group interactions, we not only reproduced the result reported by Maloney et al. (2011), finding a larger distance effect in the more anxious group, but also found a larger size effect in HMA than in LMA individuals.

As for ERPs, a positive component of larger amplitude was found in HMA individuals as compared with their LMA counterparts. Interestingly, this component was found for both the numerical distance and size effects, suggesting that HMA individuals have a less precise representation of numerical magnitude in the mental number line than do their LMA peers. To our knowledge, this is the first time that this component has been studied in high and low math-anxious individuals, and it is also the first time that it has been linked to the size effect. In the present experiment, differences between groups in the ERP distance effect were found at fronto-central positions and not at posterior electrodes. Two explanations can be given for this. First, we used a simultaneous comparison task (i.e., telling which of two simultaneously presented numbers has the larger numerical magnitude) instead of the comparison task with a fixed standard, in which the P2p component has mainly been reported (Dehaene, 1996; Libertus et al., 2007; Pinel et al., 
2001; Temple \& Posner, 1998; Turconi, 2004). Some studies using a simultaneous comparison task have failed to find a clear parietal topography for the distance effect (Szücs \& Soltész, 2008; Szűcs \& Soltész, 2007). Moreover, Szücs and Csépe (2005), using an addition verification task in which the distance from the correct solution was manipulated (they presented either the correct solution or an incorrect one that deviated \pm 2 or \pm 9 from the correct one), reported that "contrary to previous observations, the distance effect was not restricted to the parietal electrodes but it was seen over nearly all frontal, central and parietal electrodes” (p. 295). This discrepancy in the topography of the ERP distance effect is not surprising if we take into account that Maloney et al. (2010) did not find a significant correlation in response time between these two tasks (i.e., a symbolic lower/higher than 5 and a symbolic simultaneous comparison task), which suggests that the two tasks may rely on different mechanisms. Therefore, ERP generators may be different in these two tasks, and this would account for different scalp topographies.

The second explanation for our results regarding the ERP distance effect is as follows. Szücs, Soltész, Jármi, and Csépe (2007) used a simultaneous comparison task with children and adults and found that between $180 \mathrm{~ms}$ and $240 \mathrm{~ms}$ the topography of the distance effect differed in Grade 3 children relative to Grade 5 children and adults, in such a way that amplitudes were more positive frontally in the former. This effect is similar to the one we observed in the sense that the ERP distance effect of our high math-anxious individuals also differed from that of their low math-anxious peers at fronto-central positions. It is noteworthy that distance and size effects, both in response time and hit rate, are larger in children than in adults (Holloway \& Ansari, 2009; Laski \& Siegler, 2007; Sasanguie, De Smedt, Defever, \& Reynvoet, 2012), suggesting that the mental number line becomes more precise with development. Further research is needed in order to study these similarities 
between children and high math-anxious individuals when they are engaged in numerical comparison tasks.

The results of the present experiment have important implications in terms of explaining why math anxiety is related to low performance in mathematical tasks. Two arguments can be put forward. First, according to the triple-code model of number processing (Dehaene, 1997; Dehaene, Dehaene-Lambertz, \& Cohen, 1998) there are three distinct systems of number representation: a quantity system, a verbal system, and a visual system. In the quantity system, nonverbal semantic representations of numbers are represented in a continuous line (the mental number line). This system deals with the size and distance relations between numbers (Brannon, 2006), and therefore it is engaged in numerical comparison tasks. This non-verbal representation of quantity is suggested to serve as a foundation for the construction of higher-order arithmetical and mathematical concepts. Thus, the worse representation of numerical quantity found in HMA individuals could explain their deficit in the foundations of higher mathematical abilities. Second, there is evidence that the size of the numerical distance effect is related to the level of proficiency in math tasks (De Smedt et al., 2009; Holloway \& Ansari, 2009; Linsen, Verschaffel, Reynvoet, \& De Smedt, 2014; Sasanguie, De Smedt, Defever, \& Reynoet, 2012). De Smedt et al. (2009) found that more efficient math task solvers showed smaller distance effects (and therefore, their representation of numbers was more precise). Holloway and Ansari (2009) have demonstrated that the numerical distance effect decreases with age, suggesting that magnitude representation becomes increasingly more precise with increasing age (and, hence, with increasing formal mathematical education). Sasanguie et al. (2012) also observed an association between the distance effect and mathematics achievement in a symbolic number comparison task, which was strong in kindergarteners but reduced in 
older children. Linsen et al. (2014) demonstrated that children who were faster in comparing two numbers were also faster in solving mental multi-digit subtractions. These results support the idea that a poor mental representation of number, as indexed by a large distance effect, is related to a low level of math ability.

The results of the present study also have important implications in terms of how to design intervention programs to prevent and treat math anxiety (see also Maloney \& Beilock, 2012, and Maloney, Schaeffer, \& Beilock, 2013, for two reviews on this topic). As for prevention, if high math-anxious individuals suffer from a deficit of basic numerical understanding that prevents them from developing more complex mathematical skills, one way of avoiding this problem could be to focus more heavily on these basic numerical concepts in the first years of formal education. Educators should take this into account when developing their curricula so as to prevent their students from developing math anxiety. Another issue is how to help people that currently suffer from math anxiety. In other words, how might math anxiety be treated in individuals who have already developed it? In our view, it would be worthwhile developing intervention programs that include training in basic numerical concepts (such as the distance between numbers). The main objective of these programs would be to increase the precision of number representation in the mental number line for high math-anxious individuals by reducing the overlapping of mental representations of close numbers. It remains to be demonstrated, however, whether an intervention program of this kind would improve math achievement in HMA individuals.

In sum, the current results demonstrate the relationship between math anxiety and the numerical distance and size effects, as indexed by behavioral and ERP measures. Our findings provide new support for a deficit in basic processing of numerical magnitude in 
high math-anxious individuals, a deficit that involves the precision of their mental number line. These results have important implications for the teaching and remediation of math anxiety, suggesting that potentially useful intervention programs could be designed by focusing on improving the poor magnitude representation suffered by high math-anxious individuals. 


\section{REFERENCES}

Alexander, L. \& Martray, C. (1989). The development of an abbreviated version of the Mathematics Anxiety Rating Scale. Measurement and Evaluation in Counseling and Development., 22, 143-150.

Antell, S. E., \& Keating, D. P. (1983). Perception of numerical invariance in neonates. Child Development, 54, 695-701.

Ashcraft, M. H., \& Kirk, E. P. (2001). The relationships among working memory, math anxiety, and performance. Journal of Experimental Psychology. General, 130(2), 22437.

Ashcraft, M. H., \& Krause, J. A. (2007). Working memory, math performance, and math anxiety. Psychonomic Bulletin \& Review, 14(2), 243-8.

Ashcraft, M. H. \& Ridley, K. (2005). Math anxiety and its cognitive consequences: A tutorial review. In J. I. D. Campbell (Ed.), Handbook of mathematical cognition. (Psychology Press, pp. 315-325). New York.

Ashcraft, M. H., Kirk, E. P., \& Hopko, D. (1998). On the cognitive consequences of mathematics anxiety. In C. Donlan (Ed.), The development of mathematical skills (Psychology Press, pp. 175-196). East Sussex.

Brannon, E. M. (2006). The representation of numerical magnitude. Current Opinion in Neurobiology, 16(2), 222-9. 
Buckley, P. B., \& Gillman, C. B. (1974). Comparisons of digits and dot patterns. Journal of experimental psychology , 103 (6), 1131-6

De Smedt, B., Verschaffel, L., \& Ghesquière, P. (2009). The predictive value of numerical magnitude comparison for individual differences in mathematics achievement. Journal of Experimental Child Psychology, 103(4), 469-79.

Dehaene, S. (1996). The organization of brain activations in number comparison: Eventrelated potentials and the additive-factors method. Journal of Cognitive Neuroscience, 8(1), 47-68.

Dehaene, S. (1997). The number sense. New York: Oxford University Press.

Dehaene, S., \& Changeux, J. P. (1993). Development of elementary numerical abilities: a neuronal model. Journal of Cognitive Neuroscience, 5(4), 390-407.

Dehaene, S., Dehaene-Lambertz, G., \& Cohen, L. (1998). Abstract representations of numbers in the animal and human brain. Trends in Neurosciences, 21(8), 355-61.

Dehaene, S., Dupoux, E., \& Mehler, J. (1990). Is numerical comparison digital? Analogical and symbolic effects in two-digit number comparison. Journal of Experimental Psychology. Human Perception and Performance, 16(3), 626-41.

Duncan, E. M., \& McFarland, C. E. (1980). Isolating the effects of symbolic distance and semantic congruity in comparative judgments: An additive-factors analysis. Memory and Cognition, 8, 612-622.

Eysenck, M. W. (1992). Anxiety: The cognitive perspective. Hove, UK: Erlbaum. 
Eysenck, M. W. (1997). Anxiety and cognition: A unified theory. Hove, UK: Psychology Press.

Eysenck, M. W., \& Calvo, M. G. (1992). Anxiety and Performance: The Processing Efficiency Theory. Cognition \& Emotion, 6(6), 409-434.

French, J. W., Ekstrom, R.B., \& Price, L. A. (1963). Manual for kit of reference tests for cognitive factors. (E. T. Service., Ed.). Princeton, NJ.

Goetz, T., Bieg, M., Lüdtke, O., Pekrun, R., \& Hall, N. C. (2013). Do girls really experience more anxiety in mathematics? Psychological Science, 24(10), 2079-2087.

Holloway, I. D., \& Ansari, D. (2009). Mapping numerical magnitudes onto symbols: the numerical distance effect and individual differences in children's mathematics achievement. Journal of Experimental Child Psychology, 103(1), 17-29.

Hyde, D. C., \& Spelke, E. S. (2008). All numbers are not equal: An electrophysiological investigation of small and large number representations. Journal of Cognitive Neuroscience, 21(6), 1039-1053.

Hyde, D. C., \& Spelke, E. S. (2012). Spatiotemporal dynamics of processing nonsymbolic number: an event-related potential source localization study. Human Brain Mapping, 33(9), 2189-203.

Hyde, D. C., \& Wood, J. N. (2011). Spatial attention determines the nature of nonverbal number representation. Journal of Cognitive Neuroscience, 23(9), 2336-51. 
Keselman, H. J. (1998). Testing treatment effects in repeated measures designs: an update for psychophysiological researchers. Psychophysiology, 35(4), 470-8.

Koechlin, E., Naccache, L., Block, E., \& Dehaene, S. (1999). Primed numbers: Exploring the modularity of numerical representations with masked and unmasked semantic priming. Journal of Experimental Psychology: Human Perception and Performance, 25(6), 1882-1905.

Lasky, E. V., \& Siegler, R. S. (2007). Is 27 a big number? Correlational and causal connections among numerical categorization, number line estimation, and numerical magnitude comparison. Child Development, 78, 1723-1743.

Libertus, M. E., \& Brannon, E. M. (2010). Stable individual differences in number discrimination in infancy. Developmental Science, 13(6), 900-6.

Libertus, M. E., Woldorff, M. G., \& Brannon, E. M. (2007). Electrophysiological evidence for notation independence in numerical processing. Behavioral and Brain Functions, 3(1), 1-15.

Linsen, S., Verschaffel, L., Reynvoet, B., \& De Smedt, B. (2014). The association between children's numerical magnitude processing and mental multi-digit subtraction. Acta Psychologica, 145, 75-83.

Maloney, E. A., Ansari, D., \& Fugelsang, J. A. (2011). The effect of mathematics anxiety on the processing of numerical magnitude. Quarterly Journal of Experimental Psychology (2006), 64(1), 10-6. 
Maloney, E. A., \& Beilock, S, L. (2012). Math anxiety: who has it, why it develops, and how to guard against it. Trends in Cognitive Sciences, 16(8), 404-406.

Maloney, E. A., Risko, E. F., Ansari, D., \& Fugelsang, J. A. (2010). Mathematics anxiety affects counting but not subitizing during visual enumeration. Cognition, 114(2), 2937.

Maloney, E. A., Risko, E. F., Preston, F., Ansari, D., \& Fugelsang, J. (2010). Challenging the reliability and validity of cognitive measures: The case of the numerical distance effect. Acta Psychologica, 134, 154-161.

Maloney, E. A., Schaeffer, M. W., and Beilock, S. L. (2013). Mathematics anxiety and stereotype threat: shared mechanisms, negative consequences and promising interventions. Research in Mathematics Education, 15(2), 115-128.

Moyer, R. S., \& Landauer, T. K. (1967). Time required for Judgements of Numerical Inequality. Nature, 215(5109), 1519-1520.

Núñez-Peña, M. I., Suárez-Pellicioni, M., Guilera, G., \& Mercadé-Carranza, C. (2013). A Spanish version of the short Mathematics Anxiety Rating Scale (sMARS). Learning and Individual Differences, 24, 204-210.

Pinel, P., Dehaene, S., Rivière, D., \& LeBihan, D. (2001). Modulation of parietal activation by semantic distance in a number comparison task. NeuroImage, 14(5), 1013-26.

Restle, F. (1970). Speed of adding and comparing numbers. Journal of Experimental Psychology, 83, 274-278. 
Richardson, F. C., \& Suinn, R. M. (1972). The mathematics anxiety rating scale: Psychometric data. Journal of Counceling Psychology, 19(6), 551-554.

Sasanguie, D., De Smedt, B., Defever, E., \& Reynvoet, B. (2012). Association between basic numerical abilities and mathematics achievement. British Journal of Developmental Psychology, 30, 344-357.

Smets, K., Gebuis, T., \& Reynvoet, B. (2013). Comparing the neural distance effect derived from the non-symbolic comparison and the same-different task. Frontiers in Human Neuroscience, 7, 1-9.

Spielberger, C. D., Gorsuch, R., \& Lushene, R. (2008). Cuestionario de ansiedad EstadoRasgo, STAI. (TEA Ediciones). Madrid.

Spielberger, C. D., Gorsuch, R., Lushene, R., Vagg, P. R., \& Jacobs, G. A. (1983). Manual for the State-Trait anxiety inventory. (Consulting.). Palo Alto, CA.

Strauss, M. S., \& Curtis, L. E. (1981). Infant Perception of Numerocity. Child Development, 52, 1146-1152.

Szücs, D., \& Csépe, V. (2005). The effect of numerical distance and stimulus probability on ERP components elicited by numerical incongruencies in mental addition. Cognitive Brain Research, 22, 282-300.

Szücs, D., \& Soltész, F. (2007). Event-related potentials dissociate facilitation and interference effects in the numerical Stroop paradigm. Neuropsychologia, 45, 31903202. 
Szücs, D., \& Soltész, F. (2008). The interaction of task-relevant and task-irrelevant stimulus features in the number/size congruency paradigm: An ERP study. Brain Research, 1190, 143-158.

Szücs, D., Soltész, F., Jármi, E., \& Csépe, V. (2007). The speed of magnitude processing and executive functions in controlled and automatic number comparison in children: an electro-encephalography study. Behavioral and Brain Functions, 3(23), 1-20.

Temple, E., \& Posner, M. I. (1998). Brain mechanisms of quantity are similar in 5-year-old children and adults. Proceedings of the National Academy of Sciences of the United States of America, 95(13), 7836-41.

Van Selst, M., \& Jolicoeur, P. (1994). A solution to the effect of sample size and skew on outlier elimination. Quarterly Journal of Experimental Psychology. 47A, 631-650. 
Table 1. Means and standard errors of the mean (SEM; in brackets) for age, educational level, math anxiety, trait anxiety, and arithmetic ability, as well as frequencies for gender and hand dominance for the LMA and HMA groups.

\begin{tabular}{ccccccccc}
\hline & Age & Gender & Dominance & Education & sMARS & STAI-T & Ability-1 & Ability-2 \\
\hline LMA & $21.59(.63)$ & 67 & 96 & $13.48(.66)$ & $44.26(1.38)$ & $19.52(1.87)$ & $.90(.03)$ & $.24(.02)$ \\
HMA & $20.92(.30)$ & 88 & 88 & $13.50(.53)$ & $85.08(1.25)$ & $23.08(2.14)$ & $.88(.02)$ & $.19(.01)$ \\
\hline
\end{tabular}

Note: LMA: low math-anxious; HMA: high math-anxious; Gender: percentage of women; Dominance: percentage of right-handed; Education: number of years of formal education from 12 years-old onwards. sMARS: Shortened Math Anxiety Rating Scale; STAI-T: Trait anxiety subscale from the STAI. Ability-1: arithmetic ability measured taking the number of correctly solved operations over "the total number of operations attempted by the participant" on the Addition test from the French kit (i.e., a measure of precision). Ability-2: arithmetic ability measured taking the number of correctly solved operations over "the total number of operations included in the Addition test" from the French kit (i.e., a measure of speed). 
Table 2. Means and standard errors (in brackets) for response time (in ms) and hit rate (in \%) for the small and large size conditions in the LMA and HMA groups.

\begin{tabular}{lcccc}
\hline & \multicolumn{2}{c}{ Small size } & \multicolumn{2}{c}{ Large size } \\
\hline LMA & Response time & Hit rate & Response time & Hit rate \\
HMA & $344(7.9)$ & $93(1.2)$ & $374(11.8)$ & $75(2.5)$ \\
\hline
\end{tabular}


Table 3. Means and standard errors (in brackets) for response time (in ms) and hit rate (in \%) for the distance1 and distance-7 conditions in the LMA and HMA groups.

\begin{tabular}{lcccc}
\hline & \multicolumn{2}{c}{ Distance 1 } & \multicolumn{2}{c}{ Distance 7 } \\
\hline & Response time & Hit rate & Response time & Hit rate \\
\hline LMA & $374(11.8)$ & $75(2.5)$ & $329(6.5)$ & $96(0.9)$ \\
HMA & $407(12.1)$ & $77(2.6)$ & $347(6.7)$ & $95(0.9)$ \\
\hline
\end{tabular}




\section{CAPTIONS}

Figure 1. (A) Difference waves obtained after subtracting small number trial ERPs from large number trial ERPs in the LMA (thin line) and HMA (thick line) groups at Fz, Cz, and Pz. (B) Spatial distribution of the size effect over all electrodes at the scalp surface in the LMA group (right) and the HMA group (left). Large minus small number trial voltage differences in the 200-250 ms window.

Figure 2. (A) Difference waves obtained after subtracting distance-7 trial ERPs from distance-1 trial ERPs in the LMA (thin line) and HMA (thick line) groups at $\mathrm{Fz}, \mathrm{Cz}$, and Pz. (B) Spatial distribution of the distance effect over all electrodes at the scalp surface in the LMA group (right) and the HMA group (left). Distance-1 minus distance-7 trial voltage differences in the 200-250 ms window. 

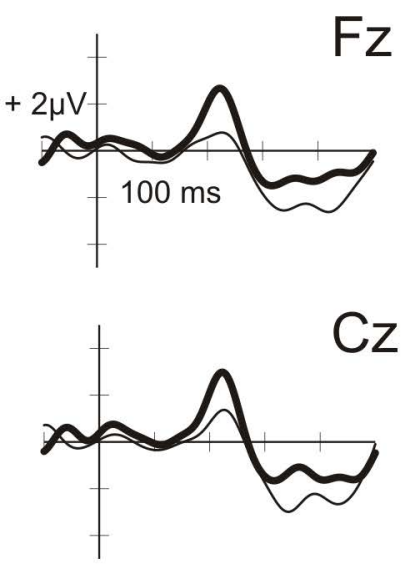

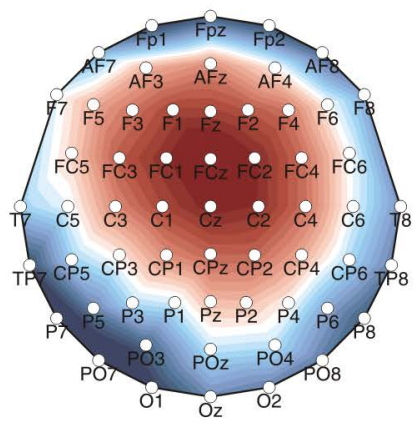

HIGH MATH ANXIETY

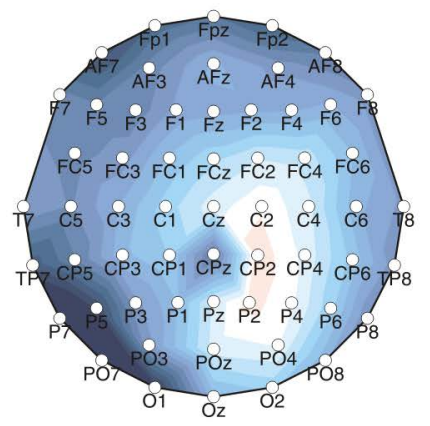

LOW MATH ANXIETY

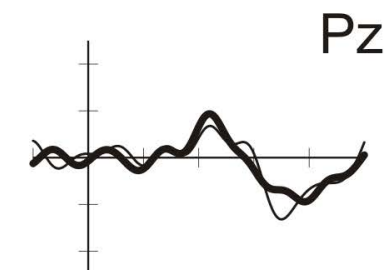

$$
+0.0 \mu \mathrm{V} \quad+2.5
$$



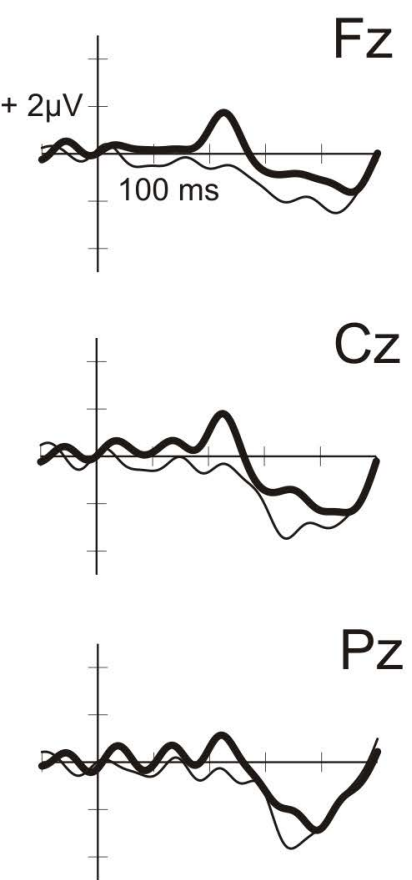

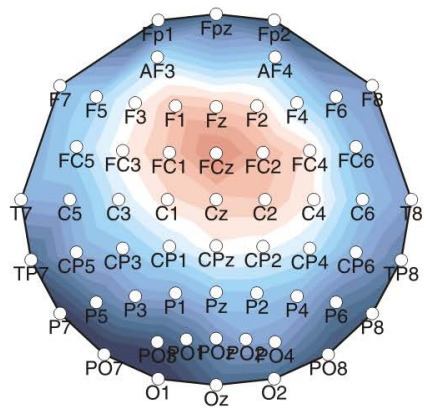

HIGH MATH ANXIETY

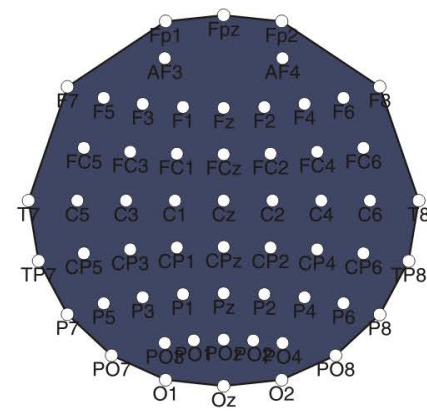

LOW MATH ANXIETY

$$
+0.0 \mu \mathrm{V} \quad+2.5
$$

